\title{
Frames and subspaces for phaseless reconstruction
}

\author{
S.Ya. Novikov, M.E. Fedina ${ }^{1}$ \\ ${ }^{1}$ Samara National Research University, Moskovskoe shosse, 34, Samara, 443086, Russia
}

\begin{abstract}
Frames and subspaces, that are used to the reconstruction of the vector signal without phase measurements, represented. The new concept of equidistributed frames is considered. The possibility of reconstruction of the vector by the norms of the projections on the subspaces is asserted. Particular attention is paid to systems of subspaces for which there is the possibility of reconstruction by the norms of the projections on them and on their orthogonal complements.
\end{abstract}

Keywords: equidistributed frames; phaseless reconstruction; complement property; full spark set; norm retrieval

\section{Basic facts. Phaseless recovery}

Let $\mathbb{H}^{M}$ denotes $M$-dimensional space with the scalar product.

Definition 1. A set of vectors $\Phi=\left\{\varphi_{k}\right\}_{k=1}^{N}$ is called a frame for the $\mathbb{H}^{M}$, if there are positive constants $A, B$ such that for all $x \in \mathbb{H}^{M}$

$$
A\|x\|^{2} \leq \sum_{k=1}^{N}\left|\left\langle x, \varphi_{k}\right\rangle\right|^{2} \leq B\|x\|^{2} .
$$

Numbers $A$ and $B$ are called the lower and upper frame bounds respectively. If we can choose $A=B$, then the frame is called tight, and if $A=B=1$, it is called a Parseval-Steklov frame (This name was proposed by Acad. V.S. Vladimirov during a report of the second author in Math. Steklov Institute in 2008 instead of usual Parseval Frame).

Note that in the finite dimensional setting, a frame is simply a spanning set of vectors in the Hilbert space $\left(\operatorname{span}\left\{\varphi_{k}\right\}_{k=1}^{N}=\mathbb{H}^{M}\right)[1,2]$.

There are three operators connected with a frame $\Phi$ : analysis operator $T: \mathbb{H}^{M} \rightarrow \ell_{2}^{N}$, defined by

$$
T(x)=\left\{\left\langle x, \varphi_{k}\right\rangle\right\}_{k=1}^{N},
$$

adjoint synthesis operator

$$
T^{*}\left(\left\{a_{k}\right\}_{k=1}^{N}\right)=\sum_{k=1}^{N} a_{k} \varphi_{k}
$$

and frame operator $S:=T^{*} T$ on $\mathbb{H}^{M}$, defined by

$$
S(x)=T^{*} T(x)=\sum_{k=1}^{N}\left\langle x, \varphi_{k}\right\rangle \varphi_{k} .
$$

The frame operator is positive, self-adjoint and invertible. Besides, we have

$$
A I \leq S \leq B I,
$$

where $I$ is identity operator in $\mathbb{H}^{M}$.

In particular, for the Parseval-Steklov frame the frame operator is the identity operator, so this frame is the most useful for the reconstruction of signals. In fact, in this case for every $x \in \mathbb{H}^{M}$ the following equality is true

$$
x=\sum_{k=1}^{N}\left\langle x, \varphi_{k}\right\rangle \varphi_{k}
$$


The operator $G=T T^{*}$ is Gram operator with the matrix

$$
\left(\begin{array}{cccc}
\left\|\varphi_{1}\right\|^{2} & \left\langle\varphi_{2}, \varphi_{1}\right\rangle, & \ldots & \left\langle\varphi_{N}, \varphi_{1}\right\rangle \\
\left\langle\varphi_{1}, \varphi_{2}\right\rangle & \left\|\varphi_{2}\right\|^{2} & \ldots & \left\langle\varphi_{N}, \varphi_{2}\right\rangle \\
\vdots & \vdots & \ddots & \vdots \\
\left\langle\varphi_{1}, \varphi_{N}\right\rangle & \left\langle\varphi_{2}, \varphi_{N}\right\rangle & \ldots & \left\|\varphi_{N}\right\|^{2}
\end{array}\right)
$$

and for the Parseval-Steklov frame coincides with the projection $P: \ell_{N}^{2} \rightarrow \ell_{N}^{2}$ to the image of the analysis operator [1, 2].

An easy way is known to construct Parseval-Steklov frames. It is based on the following proposition.

Proposition 1. Let $\left\{\varphi_{k}\right\}_{k=1}^{N}$ be a frame for $\mathbb{H}^{M}$ with bounds $A$ and B, and let $P$ be the orthogonal projection in $\mathbb{H}^{M}$ on the subspace W. Then $\left\{P \varphi_{k}\right\}_{k=1}^{N}$ is a frame for $W$ with bounds $A$ and B. In particular, if $\left\{\varphi_{k}\right\}_{k=1}^{N}$ is Parseval-Steklov frame for $\mathbb{H}^{M}$ and $P$ is the orthogonal projection on $W$, then $\left\{P \varphi_{k}\right\}_{k=1}^{N}$ is Parseval-Steklov frame for W.

Proof. We have for $x \in W$

$$
\begin{aligned}
& A\|x\|^{2}=A\|P x\|^{2} \leq \sum_{k=1}^{N}\left|\left\langle P x, \varphi_{k}\right\rangle\right|^{2}= \\
& =\sum_{k=1}^{N}\left|\left\langle x, P \varphi_{k}\right\rangle\right|^{2} \leq B\|P x\|^{2}=B\|x\|^{2} .
\end{aligned}
$$

Corollary 1. Let $\left\{e_{k}\right\}_{k=1}^{M}$ be an orthonormal basis $(O N B)$ in $\mathbb{H}^{M}$, and let $P$ be the orthogonal projection on the subspace W. Then $\left\{P e_{k}\right\}_{k=1}^{M}$ is Parseval-Steklov frame for $W$.

Corollary 1 is the foundation of the following algorithm for construction of Parseval-Steklov frame. We construct $N \times N$ unitary matrix for $N \geq M$, then we choose any $M$ rows, columns of thus obtaining $M \times N$-matrix form ParsevalSteklov frame in $\mathbb{H}^{M}$. If we construct from the remaining $N-M$ rows $(N-M) \times N$-matrix, then its columns are Parseval-Steklov frame in $\mathbb{H}^{N-M}$.

The following theorem, actually proved by Naimark, shows that such process is essentially the only one for constructing Parseval-Steklov frame [3].

\section{Theorem 1.}

Let $\Phi=\left\{\varphi_{k}\right\}_{k=1}^{N}$ be a frame in $\mathbb{H}^{M}$ with the analysis operator $T$, let $\left\{e_{k}\right\}_{k=1}^{N}$ be the standard basis in $\ell_{N}^{2}$, let $P: \ell_{N}^{2} \rightarrow \ell_{N}^{2}$ be the orthogonal projection on $\operatorname{Im}(T)$.

The following assertions are equivalent:

1. $\Phi$ is Parseval-Steklov frame for $\mathbb{H}^{M}$.

2. For all $k=1, \ldots, N$ we have $P e_{k}=T \varphi_{k}$.

3. There are vectors $\left\{\psi_{k}\right\}_{k=1}^{N} \subset \mathbb{H}^{N-M}$ such that $\left\{\varphi_{k} \oplus \psi_{k}\right\}_{k=1}^{N}$ form ONB in $\mathbb{H}^{N}$.

Besides, $\left\{\psi_{k}\right\}_{k=1}^{N}$ are Parseval-Steklov frame in $\mathbb{H}^{N-M}$.

Proof.

(1) $\Leftrightarrow(2)$. As noted, the system $\left\{\varphi_{k}\right\}_{k=1}^{N}$ forms Parseval-Steklov frame iff Gram operator $T T^{*}$ coincides with the projection $P$. So (1) and (2) are equivalent according to equality $T^{*} e_{k}=\varphi_{k}$ for $k=1, \ldots, N$.

(1) $\Rightarrow(3)$. Let's put $d_{k}=e_{k}-T \varphi_{k}, k=1, \ldots, N$. According to $(2), d_{k} \in(\operatorname{Im}(T))^{\perp}$ for all $k$. For a unitary operator

$$
\Phi:(\operatorname{Im}(T))^{\perp} \rightarrow \mathbb{H}^{N-M}
$$

let's put

$$
\psi_{k}:=\Phi d_{k}, k=1, \ldots, N
$$

We have using the isometry of the operator $T$,

$$
\left\langle\varphi_{i} \oplus \psi_{i}, \varphi_{k} \oplus \psi_{k}\right\rangle=\left\langle\varphi_{i}, \varphi_{k}\right\rangle+\left\langle\psi_{i}, \psi_{k}\right\rangle=
$$




$$
=\left\langle T \varphi_{i}, T \varphi_{k}\right\rangle+\left\langle d_{i}, d_{k}\right\rangle=\delta_{i k}
$$

(3) $\Rightarrow$ (1). Let's apply corollary 1.

As in [4], we call vectors $\left\{\psi_{k}\right\}_{k=1}^{N}$ Naimark complement of the frame $\Phi$.

For Parseval-Steklov frame, written as $\left\{P e_{k}\right\}_{k=1}^{N}$, Naimark complement is the system of vectors $\left\{(I-P) e_{k}\right\}_{k=1}^{N}$.

Naimark complements are defined only for Parseval-Steklov frames, and they are defined up to unitary equivalence. If $\left\{\varphi_{k}\right\}_{k=1}^{N} \subset \mathbb{H}_{M}$ and $\left\{\psi_{k}\right\}_{k=1}^{N} \subset \mathbb{H}^{N-M}$ complement each other, $U$ and $V$ are unitary operators $\left(U^{*} U=U U^{*}=I\right)$, then $\left\{U \varphi_{k}\right\}_{k=1}^{N},\left\{V \psi_{k}\right\}_{k=1}^{N}$ also complement each other.

An important application of frames is the reconstruction of a signal with incomplete data. In particular, much attention is attracted to the problem of the reconstruction phase information. In recent papers on this topic two aspects of the problem were emphasized: phaseless reconstruction and phase retrieval [7]. This paper focuses on the first aspect.

Definition 2. The set of vectors $\Phi=\left\{\varphi_{i}\right\}_{i=1}^{N}$ in $\mathbb{R}^{M}$ (or $\mathbb{C}^{M}$ ) provides phaseless reconstruction (PLR), if equalities of measurement modules

$$
\left|\left\langle x, \varphi_{i}\right\rangle\right|=\left|\left\langle y, \varphi_{i}\right\rangle\right|, \quad x, y \in \mathbb{R}^{M}\left(\mathbb{C}^{M}\right), \quad i=1, \ldots, N,
$$

imply the equality of vectors-signals up to unimodular factor, i.e. $x=c y$ with some $c= \pm 1$ for $\mathbb{R}^{M}$ or $c \in \mathbf{T}$ for $\mathbb{C}^{M}$, where $\mathbf{T}$ is the unit circle in $\mathbb{C}$.

In the rest of the text sets, which are satisfied the definition of 2, is called PLR-systems or PLR-sets. The next property is important in these questions.

Definition $3[4,5]$. The set $\Phi=\left\{\varphi_{n}\right\}_{n=1}^{N}$ in $\mathbb{H}^{M}$ has complement property $(C P)$, if for any $S \subseteq\{1, \ldots, N\}\left\{\varphi_{n}\right\}_{n \in S}$ or $\left\{\varphi_{n}\right\}_{n \in S^{c}}$ is complete in $\mathbb{H}^{M}$. Complement property in $\mathbb{R}^{M}$ is equivalent to PLR (theorem 2 below).

Definition $4[4,5,6]$. The spark of the set $\Phi=\left\{\varphi_{n}\right\}_{n=1}^{N} \subset \mathbb{H}^{M}$ is the cardinality of the smallest linear dependent subset of $\Phi$. If $\operatorname{spark}(\Phi)=M+1$, then any subset with $M$ vectors linear independent, in thus case $\Phi$ is called full spark set.

In earlier works the term "girth" was used instead of the term "spark". Spark of the linear independent system, for example, basic, is assumed to be zero.

Theorem $2[5,8]$.

Frame $\left\{\varphi_{n}\right\}_{n=1}^{N}$ in $\mathbb{R}^{M}$ is the PLR-system iff it has complement property. In particular, full spark frame with at least $2 M-1$ vectors is PLR-system. If $\left\{\varphi_{n}\right\}_{n=1}^{N}$ is PLR-system in $\mathbb{R}^{M}$, then $N \geq 2 M-1$, any subset with $2 M-2$ vectors can't be PLR-system.

Generally speaking, the recovery without phases is possible not only by full spark frames. Each frame, containing $(2 M-1)$ full spark frame, will also provide recovery without phases. However, if the frame contains exactly $2 M-1$ elements, it is a PLR-system only for full spark frame $[5,8$.

If $\Phi$ is the Parseval-Steklov frame for $\mathbb{H}^{M}$ with $N$ elements, the analysis operator is isometric according to

$$
\|T x\|^{2}=\sum_{n=1}^{N}\left|\left\langle x, \varphi_{n}\right\rangle\right|^{2}=\|x\|^{2}, \quad x \in \mathbb{H}^{M} .
$$

In this case, we obtain the reconstruction identity $x=\sum_{n=1}^{N}\left|\left\langle x, \varphi_{n}\right\rangle\right| \varphi_{n}$, or $x=T^{*} T x$. In this case, we also have that the Gramian $G:=T T^{*}$ is a rank-M orthogonal projection, because $G^{*} G=T T^{*} T T^{*}=T T^{*}=G$ and the rank of $G$ equals the trace, $\operatorname{tr} G=M$.

Definition 5. Two frames $\Phi=\left\{\varphi_{n}\right\}_{n=1}^{N}$ and $\Phi^{\prime}=\left\{\varphi_{n}^{\prime}\right\}_{n=1}^{N}$ for a finite dimensional space $\mathbb{H}^{M}$ are called unitarily equivalent if there exists an orthogonal or unitary operator $U$ on $\mathbb{H}^{M}$ such that $\varphi_{n}=U \varphi_{n}^{\prime}$ for $n=1, \ldots, N$.

Each equivalence class of frames is characterized by the corresponding Gram matrix.

Proposition 2 [13]. The Gramians of two frames $\Phi=\left\{\varphi_{n}\right\}_{n=1}^{N}$ and $\Phi^{\prime}=\left\{\varphi_{n}^{\prime}\right\}_{n=1}^{N}$ for $\mathbb{H}^{M}$ are identical if and only if the frames are unitarily equivalent. 
In [13] the new class of frames is introduced.

Definition 6. Let $\Phi=\left\{\varphi_{n}\right\}_{n=1}^{N}$ be Parseval-Steklov frame for $\mathbb{H}^{M}$, let $G$ be its Gramian. The frame $\Phi$ is called equidistributed if for each pair $p, q \in \mathbb{Z}_{N}$ there exists a permutation $\pi$ on $\mathbb{Z}_{N}$ such that $\left|G_{j, p}\right|=\left|G_{\pi(j), q}\right|$ for all $j \in \mathbb{Z}_{N}$.

In other words, $\Phi$ is equidistributed if and only if the magnitudes in any column of the Gram matrix repeat in any other column, up to a permutation of their position.

Proposition 3. If $\Phi=\left\{\varphi_{n}\right\}_{n=1}^{N}$ is an equidistributed Parseval-Steklov frame in $\mathbb{H}^{M}$, then $\left\|\varphi_{n}\right\|^{2}=M / N, n=$ $1,2, \ldots, N$.

Proof. By assumption, for each $n$ there exists $\pi$ such that $\left|G_{n, p}\right|=\left|G_{\pi(n), 1}\right|$ holds for the entries of the associated Gram matrix $G$ for all $n$. By the Parseval-Steklov identity

$$
\left\|\varphi_{p}\right\|^{2}=\sum_{n=1}^{N}\left|\left\langle\varphi_{p}, \varphi_{n}\right\rangle\right|^{2}=\sum_{n=1}^{N}\left|G_{n, p}\right|^{2}=\sum_{n=1}^{N}\left|G_{\pi(n), 1}\right|^{2}=\left\|\varphi_{1}\right\|^{2} .
$$

The trace condition $\sum_{n=1}^{N} G_{n, n}=\sum_{n=1}^{N}\left\|\varphi_{p}\right\|^{2}=M$ for the Gram matrices of Parseval-Steklov frames implies that $\left\|\varphi_{n}\right\|^{2}=M / N, n=1,2, \ldots, N$.

\section{Examples:}

1. Equiangular Parseval-Steklov frames.

Let $\Phi=\left\{\varphi_{n}\right\}_{n=1}^{N}$ be an equal-norm frame and there exists $C \geq 0$ such that $\left|\left\langle\varphi_{n}, \varphi_{n}^{\prime}=C\right\rangle\right|$ for all $n, n^{\prime} \in \mathbb{Z}_{N}$ with $n \neq n^{\prime}$. Such frames are called equiangular. Such Parseval-Steklov frames exist only with some restrictions on $N$ and $M$ [13]. The simplest example of the equiangular Parseval-Steklov frame in $\mathbb{R}^{2}$ is a well-known "Mercedes-Benz frame".

Magnitudes of the entries of any column of $G$ for such frame consist of $N-1$ instances of $C$ and one instance of $M / N$, so $\Phi$ is equidistributed.

\section{Mutually unbiased bases.}

Such frame is union of orthonormal bases such that the modulus of the inner product between any two vectors from distinct bases is constant. Such examples are widely used in quantum information theory. The simplest example of mutually unbiased bases in $\mathbb{C}^{2}$ is given by the following three bases:

$$
M_{0}=\left(\begin{array}{ll}
1 & 0 \\
0 & 1
\end{array}\right), \quad M_{1}=\frac{1}{\sqrt{2}}\left(\begin{array}{cc}
1 & 1 \\
1 & -1
\end{array}\right), \quad M_{2}=\frac{1}{\sqrt{2}}\left(\begin{array}{cc}
1 & 1 \\
i & -i
\end{array}\right) .
$$

To get the Parseval-Steklov frames one should renorm vectors to $\Phi$ because of these 3 matrices must the multiplied to $1 / \sqrt{3}$. We get the Gram matrix

$$
G=\left(\begin{array}{cccccc}
\frac{1}{3} & 0 & \lambda & \lambda & \lambda & \lambda \\
0 & \frac{1}{3} & \lambda & -\lambda & -i \lambda & i \lambda \\
\lambda & \lambda & \frac{1}{3} & 0 & \frac{1-i}{6} & \frac{1+i}{6} \\
\lambda & -\lambda & 0 & \frac{1}{3} & \frac{1+i}{6} & \frac{1-i}{6} \\
\lambda & i \lambda & \frac{1+i}{6} & \frac{1-i}{6} & \frac{1}{3} & 0 \\
\lambda & -i \lambda & \frac{1-i}{6} & \frac{1+i}{6} & 0 & \frac{1}{3}
\end{array}\right),
$$

where $\lambda=\sqrt{2} / 6$.

\section{Group frames.}

Let $\Gamma$ be a finite group of size $N=|\Gamma|$ and $\pi: \Gamma \rightarrow B\left(\mathbb{H}^{M}\right)$ be an orthogonal or unitary representation of $\Gamma$ on the real or complex space $\mathbb{H}^{M}$ respectively.

The orbit $\Phi=\left\{f_{g}=\pi(g) f_{e}\right\}_{g \in \Gamma}$, generated by a vector $f_{e}$ of norm $\sqrt{N / M}$, indexed by the unit $e$ of the group, forms the Parseval-Steklov frame, if the representation is irreducible [14].In this case $\Phi$ is equidistributed, because $\left\langle f_{g}, f_{h}\right\rangle=\left\langle\pi\left(h^{-1} g\right) f_{e}, f_{e}\right\rangle$, and left multiplication $h_{y} h^{-1}$ acts as a permutation on the group elements. So the entries of Gram matrix has equal modules, up to a permutation in rows (columns).

\section{Cycle frames.}


Consider the Discrete Fourier Transform matrix

$$
F=\frac{1}{\sqrt{N}}\left(\omega^{j l}\right)_{j, l=1}^{N}, \quad \text { where } \omega=e^{\frac{2 \pi i}{N}} .
$$

Its columns form an orthonormal basis for $\mathbb{C}^{N}$. If $A$ is a $M \times N$ matrix obtained by deleting any choice of $N-M$ rows from $F$, then its columns form a Parseval-Steklov frame for $\mathbb{C}^{N}$.

Definition 7. Let $b_{1}, \ldots, b_{M} \in\{1,2, \ldots, N\}$ be any choice of distinct integers. F frame $\Phi=\left\{\varphi_{n}\right\}_{n} \in \mathbb{Z}_{N}$, where

$$
\varphi_{n}=\frac{1}{\sqrt{N}}\left(\omega^{n l_{m}}\right)_{m=1}^{N}, \quad \text { for all } n \in \mathbb{Z}_{N},
$$

is called a cycle frame.

Every cycle frame is equidistributed, and, because the construction described above works for every pair of positive integers $M$ and $N$ with $M<N$, the existence of equidistributed frames is ensured in the complex setting.

\section{Theorem 3.}

For every $N>M$ there exists equidistributed Parseval-Steklov frame in $\mathbb{C}^{M}$ with $N$ vectors.

Let's see if the possibility of recovery without phases is transferred to the Naimark complements. We require the following theorem for this.

Theorem 4 [9].

Let $P$ be an projection in $\mathbb{H}^{N}$ with $O N B\left\{e_{n}\right\}_{n=1}^{N}$ and $S \subset\{1,2, \ldots, N\}$.

The following assertions are equivalent:

1. $\left\{P e_{i}\right\}_{i \in S}$ linear independent.

2. $\operatorname{span}\left\{(I-P) e_{i}\right\}_{i \in S^{c}}=(I-P)\left(\mathbb{H}^{N}\right)$.

Proof.

$(1) \Rightarrow(2)$. Let's suppose, that

$$
\operatorname{span}\left\{(I-P) e_{i}\right\}_{i \in S^{c}} \neq(I-P)\left(\mathbb{H}^{N}\right) .
$$

It means, that there exists $0 \neq x \in(I-P)\left(\mathbb{H}^{N}\right)$ such that $x \perp \operatorname{span}\left\{(I-P) e_{i}\right\}_{i \in S^{c}}$. As $x=\sum_{i=1}^{N}\left\langle x, e_{i}\right\rangle(I-P) e_{i}$, then

$$
\left\langle x,(I-P) e_{i}\right\rangle=\left\langle(I-P) x, e_{i}\right\rangle=\left\langle x, e_{i}\right\rangle=0
$$

for any $i \in S^{c}$. Hence, $x=\sum_{i \in S}\left\langle x, e_{i}\right\rangle e_{i}$, so

$$
\sum_{i \in S}\left\langle x, e_{i}\right\rangle e_{i}=x=(I-P) x=\sum_{i \in S}\left\langle x, e_{i}\right\rangle(I-P) e_{i},
$$

i.e. $\sum_{i \in S}\left\langle x, e_{i}\right\rangle P e_{i}=0$, and, thus, $\left\{P e_{i}\right\}_{i \in S}$ are linearly dependent.

$(2) \Rightarrow(1)$. Let's suppose, that $\left\{P e_{i}\right\}_{i \in S}$ are linearly dependent: there exist numbers $\left\{b_{i}\right\}_{i \in S}$, among which there are nonzero, and $\sum_{i \in S} b_{i} P e_{i}=0$. Then

$$
x:=\sum_{i \in S} b_{i}(I-P) e_{i}=\sum_{i \in S} b_{i} e_{i} \in(I-P)\left(\mathbb{H}^{N}\right) .
$$

Let's consider

$$
\left\langle x,(I-P) e_{j}\right\rangle=\left\langle(I-P) x, e_{j}\right\rangle=\left\langle\sum_{i \in S} b_{i} e_{i}, e_{j}\right\rangle=\sum_{i \in S} b_{i}\left\langle e_{i}, e_{j}\right\rangle=0
$$


if $j \in S^{c}$. Thus, $x \perp \operatorname{span}\left\{(I-P) e_{i}\right\}_{i \in S^{c}}$, and hence,

$$
\operatorname{span}\left\{(I-P) e_{i}\right\}_{i \in S^{c}} \neq(I-P)\left(\mathbb{H}^{N}\right) .
$$

Proposition 4. Parseval-Steklov frame is a full spark frame iff Naimark complement of this frame is a full spark frame also.

Proof. By theorem 1, Parseval-Steklov frame can be written as $\left\{P e_{i}\right\}_{i=1}^{N}$, where $\left\{e_{i}\right\}_{i=1}^{N}$ is an ONB in $\mathbb{H}^{N}$ and $P$ is the orthogonal projection in $\mathbb{H}^{N}$. Naimark complement for Parseval-Steklov frame looks as $\left\{(I-P) e_{i}\right\}_{i=1}^{N}$. By definition $\left\{P e_{i}\right\}_{i=1}^{N}$ is a full spark frame, if for any $S \subseteq\{1, \ldots, N\}$ with $|S|=M\left\{P e_{i}\right\}_{i \in S}$ is a basis in the range of the projection $P$. By theorem 3, we have that $\left\{(I-P) e_{i}\right\}_{i \in S^{c}}$ is a basis in the range of the projection $I-P$, so $\left\{(I-P) e_{i}\right\}_{i=1}^{N}$ is a full spark frame also. The reverse assertion is proved similarly.

If Parseval-Steklov frame ensures recovery without phases, Naimark complement can not provide recovery without phases. The thing is including, in particular, that in Naimark complement may be insufficient number of vectors.

Proposition 5. If Parseval-Steklov frame $\left\{\varphi_{n}\right\}_{n=1}^{N}$ ensures recovery without phases in $\mathbb{R}^{M}$, and Naimark complement to this frame also ensures recovery without phases in $\mathbb{R}^{N-M}$, then

$$
2 M-1 \leq N \leq 2 M+1 .
$$

Proof. If $\left\{\varphi_{n}\right\}_{n=1}^{N}$ ensures recovery without phases in $\mathbb{R}^{M}$, then $N \geq 2 M-1$ (theorem 2). If Naimark complement ensures recovery without phases in $\mathbb{R}^{N-M}$, then $N \geq 2(N-M)-1$, or $N \leq 2 M+1$.

But Naimark complement can fail to ensure recovery without phases even under conditions of proposition 3.

Example. Let $\left\{\varphi_{m}\right\}_{m=2}^{2 M}$ be the full spark frame in $\mathbb{R}^{M}, M \geq 3$. Let's put $\varphi_{1}=\varphi_{2}$, and let $S$ be the frame operator for $\left\{\varphi_{m}\right\}_{m=1}^{2 M}$. Note that $\left\{S^{-\frac{1}{2}} \varphi_{m}\right\}_{m=2}^{2 M}$ is full spark frame, and ensures recovery without phases. For any partition $\mathcal{S}, \mathcal{S}^{c} \subset\{1, \ldots, 2 M\}$ one of the sets $\mathcal{S}$ or $\mathcal{S}^{c}$ has at least $M$ elements from the full spark frame $\left\{S^{-\frac{1}{2}} \varphi_{m}\right\}_{m=2}^{2 M}$ and hence complete in $\mathbb{R}^{M}$.

Now let's show, that Naimark complement for $\left\{S^{-\frac{1}{2}} \varphi_{m}\right\}_{m=1}^{2 M}$ does not ensure recovery without phases. Let's break $\left\{S^{-\frac{1}{2}} \varphi_{m}\right\}_{m=1}^{2 M}$ on $\left\{S^{-\frac{1}{2}} \varphi_{m}\right\}_{m=1}^{2}$ and $\left\{S^{-\frac{1}{2}} \varphi_{m}\right\}_{m=3}^{2 M}$. None of them is linear independent, as $\varphi_{1}=\varphi_{2}$, and $M \geq 3$. According to theorem 3, Naimark complements for each of these sets are not comlete in $\mathbb{R}^{2 M-M}=\mathbb{R}^{M}$. Thus, there is a partition of Naimark complement which contradicts the complement property and does not ensure phaseless recovery.

If Parseval-Steklov frame is full spark frame, then phaseless recovery is inherited by Naimark complement.

Proposition 6. If $\Phi=\left\{\varphi_{n}\right\}_{n=1}^{N}$ is full spark Parseval-Steklov frame, $2 M-1 \leq N \leq 2 M+1$, then $\Phi$ ensures phaseless recovery in $\mathbb{R}^{M}$, and Naimark complement for $\Phi$ ensures phaseless recovery in $\mathbb{R}^{N-M}$.

Proof. By proposition 2 Naimark complement for $\Phi$ is full spark frame in $\mathbb{R}^{N-M}$. We have $2 M-1 \leq N$ and $2(N-M)-1 \leq N$, then, by theorem 2 , both $\Phi$ and its Naimark complement have complement property in relevant spaces.

\section{Recovery by the norms of projections}

Following $[4,10]$ we define the recovery of a vector-signal by the norms of projections on subspaces.

Definition 8. Let $\left\{W_{n}\right\}_{n=1}^{N}$ be the set of subspaces in $\mathbb{H}^{M}$, let $\left\{P_{n}\right\}_{n=1}^{N}$ be orthogonal projections on these subspaces. We say, that $\left\{W_{n}\right\}_{n=1}^{N}$ (or $\left\{P_{n}\right\}_{n=1}^{N}$ ) ensures recovery by the norms of projections, if for any $x, y \in \mathbb{H}^{M}$ equalities $\left\|P_{n} x\right\|=\left\|P_{n} y\right\|$ for $n=1, \ldots, N$ imply $x=c y$ for some $c$ with $|c|=1$.

Further such sets of subspaces will be called RNP-sets. 
A lot of attention to such recovery is paid in [10]. For one-dimensional subspace $W_{n}$ the number $\left\|P_{n} x\right\|$ can be received only from two vectors $\pm P_{n} x$. For subspaces $W_{n}$ with higher dimensions we have continuum of vectors with $\left\|P_{n} x\right\|$.

Nevertheless the map

$$
\mathcal{A}(x)(n)=\left\|P_{n} x\right\|
$$

can be injective for subspaces with higher dimensions. The proof of this result uses the scheme of [10], we need some auxiliary assertions.

\section{Lemma 1.}

Let $\left\{\varphi_{n}\right\}_{n=1}^{N}$ be full spark frame in $\mathbb{R}^{M}$. Let's define ONB in $\mathbb{R}^{M}$ using the following algorithm: $\psi_{1}$ is a random vector, $\psi_{2}$ is a random vector from $\left[\operatorname{span}\left(\psi_{1}\right)\right]^{\perp}, \ldots, \psi_{k}$ is a random vector from $\left[\operatorname{span}\left(\left\{\psi_{n}\right\}_{n=1}^{k-1}\right)\right]^{\perp}$. Then $\left\{\varphi_{n}\right\}_{n=1}^{N} \cup$ $\left\{\psi_{m}\right\}_{m=1}^{M}$ is the full spark frame with the probability 1 .

Proof.

Let $1 \leq k<M$. We suppose, that $\left\{\varphi_{n}\right\}_{n=1}^{N} \cup\left\{\psi_{m}\right\}_{m=1}^{k}$ is full spark frame, we need to check, that $\left\{\varphi_{n}\right\}_{n=1}^{N} \cup\left\{\psi_{m}\right\}_{m=1}^{k+1}$ is full spark frame too. For this we have to show that $\psi_{k+1}$ does not lie in the span of any $M-1$ vectors from $\left\{\varphi_{n}\right\}_{n=1}^{N} \cup\left\{\psi_{m}\right\}_{m=1}^{k}$. Choose any $M-1$ such vectors and denote them by $A$. Put $W_{k}:=\left[\operatorname{span}\left(\left\{\psi_{m}\right\}_{m=1}^{k}\right)\right]^{\perp}$ and pick $\psi_{k+1}$ as a random unit norm vector from this $(M-k)$-dimensional space. Then $\left\{\varphi_{n}\right\}_{n=1}^{N} \cup\left\{\psi_{m}\right\}_{m=1}^{k+1}$ is full spark system $\Leftrightarrow$ $\psi_{k+1} \notin \operatorname{span}(A)$. The last is truly with probability 1 iff

$$
\operatorname{dim}\left(\operatorname{span}(A) \cap W_{k}\right) \leq(M-k)-1 .
$$

In fact, $\operatorname{span}(A) \cap W_{k}$ is a subset in $(M-k)$-dimensional space $W_{k}$, and so inequality (1) implies that this intersection has zero measure. Hence, we have with probability $1 \psi_{k+1} \notin \operatorname{span}(A) \cap W_{k}$ and $\psi_{k+1} \in W_{k}$. Now we are going to the proof of inequality (1).

Let's apply the method of mathematical induction. A vector $\psi_{1}$ is chosen randomly from $W_{0}=\mathbb{R}^{M}$. If $A$ any $M-1$ vectors from $\left\{\varphi_{n}\right\}_{n=1}^{N}$, then

$$
\operatorname{dim}\left(\operatorname{span}(A) \cap W_{0}\right)=M-1,
$$

and $\left\{\varphi_{n}\right\}_{n=1}^{N} \cup \psi_{1}$ is full spark frame with probability 1 .

Let's suppose that $\left\{\varphi_{n}\right\}_{n=1}^{N} \cup\left\{\psi_{m}\right\}_{m=1}^{k}$ is full spark frame. We denote by $A$ any $M-1$ vectors from $\left\{\varphi_{n}\right\}_{n=1}^{N} \cup\left\{\psi_{m}\right\}_{m=1}^{k}$.

Let's consider two possible cases.

1. $\psi_{k} \notin A$. We have $W_{k} \subset W_{k-1}$ and

$$
\operatorname{span}(A) \cap W_{k}=\left(\operatorname{span}(A) \cap W_{k-1}\right) \cap W_{k} .
$$

Note that $\operatorname{dim} W_{k}=M-k, \operatorname{dim}\left(\operatorname{span}(A) \cap W_{k-1}\right) \leq M-k$, because $\psi_{k} \notin A$. So for the proof (1) it's suffice to check that these subspaces do not match. Let's suppose that $\operatorname{span}(A) \cap W_{k-1}=W_{k}$. We remember that $\psi_{k} \in W_{k}^{\perp}$, and hence, $\psi_{k} \in\left[\operatorname{span}(A) \cap W_{k-1}\right]^{\perp}$, this subspace has dimension $k$. As $\psi_{k} \notin W_{k-1}^{\perp}, \operatorname{dim} W_{k-1}=k-1$, and

$$
W_{k}^{\perp} \subset\left[\operatorname{span}(A) \cap W_{k-1}\right]^{\perp},
$$

it turns to be that $\psi_{k}$ lies in one-dimensional subspace, determined by $\operatorname{span}(A)$ and $W_{k-1}$. It's possible only with zero probability for randomly chosen vector from $M-(k-1)$-dimensional subspace $W_{k-1}$.

2. $\psi_{k} \in A$. Let's note that

$$
\operatorname{dim}\left(\operatorname{span}(A) \cap W_{k}\right) \leq M-k,
$$

as $\operatorname{dim}\left(W_{k}\right)=M-k$. For contradiction, we suppose that

$$
\operatorname{dim}\left(\operatorname{span}(A) \cap W_{k}\right)=M-k .
$$

We have further that

$$
W_{k} \subset \operatorname{span}(A) .
$$


Pick $\varphi \in\left\{\varphi_{n}\right\}_{n=1}^{N}$ so that $\varphi \notin A$. Then

$$
\operatorname{dim}\left(\operatorname{span}\left(A \backslash \psi_{k}\right) \cap W_{k}\right) \leq \operatorname{dim}\left(\operatorname{span}\left(A \backslash \psi_{k} \cup \varphi\right) \cap W_{k}\right) \leq(M-k)-1 .
$$

The last inequality is a result of the first case above.

On the other hand as $\psi_{k} \perp W_{k}$ and $\psi_{k} \in A$, we receive from (2) and (3)

$$
\operatorname{dim}\left(\operatorname{span}\left(A \backslash \psi_{k}\right) \cap W_{k}\right)=\operatorname{dim}\left(\operatorname{span}(A) \cap W_{k}\right)=M-k .
$$

This contradiction proves (1).

Corollary 3. The finite set of ONB, which are built by the algorithm of random choice of lemma 1, is full spark frame with the probability 1 .

Proof. Let's apply consistently the lemma 1.

Lemma 2. For an integer $M \geq 2$ let's pick integers $M-1 \geq I_{1} \geq I_{2} \geq \ldots \geq I_{1} \geq 1$. There is a real invertible $M \times M$-matrix with $0-1$ instances such that the $k$-row has exactly $I_{k}$ ones.

Proof.

We apply induction by $M$. The claim is obvious for $M=2$. Let's suppose that the assertion is valid for $M$. Let's look at the set of $M+1$ numbers such that

$$
M=I_{1}=\ldots=I_{s}>I_{s+1} \geq \ldots \geq I_{M+1} \geq 1
$$

for some $s \leq M+1$. By induction assumption for the set of numbers

$$
I_{1}-1=\ldots=I_{s}-1 \geq I_{s+1} \geq \ldots \geq I_{M} \geq 1
$$

there is the invertible $M \times M$-matrix $A=\left[a_{i j}\right]_{i, j=1}^{M}$ with $I_{k-1}-1=M-1$ ones in $k$-row for $k=1, \ldots, s$ and $I_{k}$ ones in $k$-row for $k=s+1, \ldots, M$. Let's define $(M+1) \times(M+1)$-matrix $B=\left[b_{i j}\right]_{i, j=1}^{M+1}$ defining

$$
b_{i j}=\left\{\begin{array}{cc}
a_{i j}, & 1 \leq i, j \leq M, \\
1, & 1 \leq i \leq s, j=M+1, \\
1, & i=M+1,1 \leq j \leq M+1, \\
0, & \text { for other indexes }
\end{array}\right.
$$

The matrix $B$ has $I_{k}$ ones in $k$-row for $k=1, \ldots, M+1$. The matrix $A=\left[a_{i j}\right]_{i, j=1}^{M}=\left[b_{i j}\right]_{i, j=1}^{M}$ is invertible, so the matrix $B$ by row reduces can be reduced to the step form $\widetilde{B}=\left[\widetilde{b}_{i j}\right]_{i, j=1}^{M+1}$, where $\left[\widetilde{b}_{i j}\right]_{i, j=1}^{M}=I_{M \times M}$, and the row $(M+1)$ is not changed. If we suppose that $\widetilde{B}$ is not invertible, then the row $(M+1)$ by row reduces can be reduced to the zero row and hence

$$
\sum_{i=1}^{I_{M+1}} \widetilde{b}_{M+1, i}=0 .
$$

Let's define for each $l \in\left\{1, \ldots, I_{M+1}\right\}$ the matrix $\widetilde{B}_{l}$. It is obtained from the matrix $\widetilde{B}$ changing $\widetilde{b}_{M+1, M+1}=0$ to $\widetilde{b}_{M+1, l}=1$.

If $\widetilde{B}$ is not invertible, then by row reduces the last row is reduced to the zero row, and we have

$$
\sum_{i=1, i \neq l}^{I_{M+1}} \widetilde{b}_{M+1, i}=-1 .
$$

The equality (6) is valid for any $l \in\left\{1, \ldots, I_{M+1}\right\}$, that's contradict to (5). Hence at least one of the matrixes $\widetilde{B}$ or $\widetilde{B}_{l}$ for some $l \in\left\{1, \ldots, I_{M+1}\right\}$ has to be invertible. 
Theorem 6. There exists $R N P$-set in $\mathbb{R}^{M}$ consisting from $2 M-1$ subspaces, dimension of each subspace $<M-1$. Proof.

Let $\left\{\varphi_{n}\right\}_{n=1}^{2 M-1}$ be the set of vectors in $\mathbb{R}^{M}$ with complement property and with additional requirement of orthogonality and normalization $(\|\cdot\|=1)$ to the sets $\left\{\varphi_{n}\right\}_{n=1}^{M}$ and $\left\{\varphi_{n}\right\}_{n=M+1}^{2 M-1}$. The corollary 2 ensures the existence of such set. Let $I_{k} \subseteq\{1, \ldots, M\}$ for $k=1, \ldots, M$, and $J_{k} \subseteq\{M+1, \ldots, 2 M-1\}$ for $k=M+1, \ldots, 2 M-1$, let $P_{I_{k}}$ and $P_{J_{k}}$ be projections on $\operatorname{span}\left(\left\{\varphi_{n}\right\}_{n \in I_{k}}\right)$ and $\operatorname{span}\left(\left\{\varphi_{n}\right\}_{n \in J_{k}}\right)$ respectively. The next construction ensures phaseless recovery for $x \in \mathbb{R}^{M}$ by $\left\|P_{I_{k}} x\right\|$ and $\left\|P_{J_{k}} x\right\|$ for $k=1, \ldots, 2 M-1$.

Let $A=\left[a_{k z}\right]_{k, z=1}^{M}$ be $M \times M$-matrix, its rows are agreed with $I_{k}$, i. e. $a_{k z}=1$, for $z \in I_{k}$, and $a_{k z}=0$ for other $z$.

Similarly we define the matrix $B=\left[b_{k z}\right]_{k, z=1}^{M-1}$ as $(M-1) \times(M-1)$-matrix with $b_{k z}=1$ for $z+M \in J_{k}$, and $b_{k z}=0$ for other $z$.

Let's look at the subspaces $\operatorname{span}\left(\left\{\varphi_{n}\right\}_{n \in I_{k}}\right)$ for $k=1, \ldots, M$. For $x \in \mathbb{R}^{M}$ we have

$$
\left\|P_{I_{k}} x\right\|^{2}=\sum_{n \in I_{k}}\left|\left\langle x, \varphi_{n}\right\rangle\right|^{2}
$$

whence

$$
\left[\begin{array}{c}
\left\|P_{I_{1}} x\right\|^{2} \\
\vdots \\
\left\|P_{I_{M}} x\right\|^{2}
\end{array}\right]=A\left[\begin{array}{c}
\left|\left\langle x, \varphi_{1}\right\rangle\right|^{2} \\
\vdots \\
\left|\left\langle x, \varphi_{M}\right\rangle\right|^{2}
\end{array}\right] .
$$

This equation may be solved upon $\left\{\left|\left\langle x, \varphi_{n}\right\rangle\right|\right\}_{n=1}^{M}$, if the matrix $A$ is invertible. Similar equation may be written with the matrix $B$. Hence if the matrixes $A$ and $B$ are invertible, we obtain the complete set of "measurements" $\left\{\left|\left\langle x, \varphi_{n}\right\rangle\right|\right\}_{n=1}^{2 M-1}$. The set $\left\{\varphi_{n}\right\}_{n=1}^{2 M-1}$ has complement property and according to theorem 2 , phaseless recovery is possible using subspaces $\operatorname{span}\left(\left\{\varphi_{n}\right\}_{n \in I_{k}}\right)$ and $\operatorname{span}\left(\left\{\varphi_{n}\right\}_{n \in J_{k}}\right)$ for $k=1, \ldots, 2 M-1$. To complete the proof we choose $\left\{I_{k}\right\}_{k=1}^{M}$ and $\left\{J_{k}\right\}_{k=M+1}^{2 M-1}$ to provide the invertibility of the matrixes $A$ and $B$.

Let's note that the quantity of ones in each row coincides with the dimension of the appropriate subspace. Such selection is possible according to lemma 2 for any subsets $I_{k}, J_{k}$, with $1 \leq\left|I_{k}\right| \leq M-1$ and $1 \leq\left|J_{k}\right| \leq M-2$.

The answer to the next question is unknown [4]:

Question. Is it possible phaseless recovery by norms of projections in $\mathbb{R}^{M}$ with the set of subspaces $\left\{W_{n}\right\}_{n=1}^{N}$ for $N<2 M-1$ ?

\section{Acknowledgements}

The first author was supported by RFBR grant N 7-01-00138.

\section{References}

[1] Novikov SYa, Likhobabenko MA. Frames in finite-dimensional spaces. Samara: Samarsky Universitet, 2013; 52 p.

[2] Christensen O. An Introduction to Frames and Riesz bases. Boston: Birkh auser, 2003; 403 p.

[3] Kashin BS, Kulikova TYu. A note about Frames. Matem. zametki 2002; 72(6): 941-945.

[4] Bahmanpour S, Cahill J, Casazza PG, Jasper J, Woodland LM. Phase retrieval and norm retrieval. URL : https://arxiv.org/abs/1409.8266.

[5] Bandeira AS, Cahill J, Mixon G, Nelson AA. Saving phase: Injectivity and stability. URL: https://arxiv.org/abs/1302.4618v1.

[6] Alexeev B, Cahill J, Mixon DG. Full Spark Frames. URL: https://arxiv.org/abs/1110.3548.

[7] Botelho-Andrade S, Casazza PG, Nguyen HV, Tremain JC. Phase retrieval verses phaseless reconstruction. URL: https://arxiv.org/abs/1507.05815.

[8] Novikov SYa, Fedina ME. Restoring the signal be modules of measurement. Vestnik Samara University 2016; 3-4: 63-74.

[9] Bodmann B, Casazza PG, Paulsen V, Speegle D. Spanning and independence properties of frame partitions. Proc. Am. Math. Soc. 2012; 140(7): $2193-2207$.

[10] Cahill J, Casazza PG, Peterson J, Woodland LM. Phase retrieval by projections. URL: https://arxiv.org/abs/1305.6226.8858.

[11] Horn R, Johnson Ch. Matrix analysis. Moskva: Mir, 1989; 655 p.

[11] Cahill J, Chen X. A note on scalable frames. URL: https://arxiv.org/abs/1301.7292.

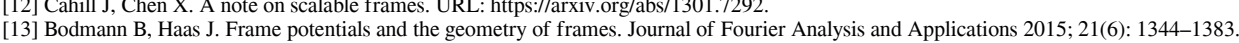

[14] Vale R, Waldron S. Tight frames and their symmetries. Constructive Approximation 2004; 21(1): 83-112. 